Wojciech Misztal

Pontifical University of John Paul II in Cracow, Poland

\title{
Dans le Christ Jésus: \\ l'oeuvre du salut comme la réalisation de la communion dans les dimensions verticale et horizontale
}

\section{In Christ Jesus: the Mystery of Salvation as Accomplishment of the Unity in its Dimensions Vertical and Horizontal}

\begin{abstract}
The theme "In Christ Jesus: the mystery of salvation as accomplishment of the unity in its dimensions vertical and horizontal" will concern these following points: 1) redemption as multiple realization of God's blessing, as multiple realization of paternity; 2) the Son of God and the mystery of adoption of the creature beings; 3) the divine gift of the communion in Christ; 4) the accomplishment of God's and creature's purpose: the concept of the new creation.
\end{abstract}

\section{Keywords}

Bible, Christianity, Church, creation, God, human being, Jesus Christ, salvation, spirituality, theology.

En présentant dans l'Épître aux Galates sa compréhension du christianisme - c'est-à-dire de la vie chrétienne, de l'expérience et de l'espérance qui sont propres aux chrétiens - st Paul Apôtre parle du Christ, de son œuvre, de sa place, de son rôle dans le mystère de la création-rédemption. Dans la Lettre aux Galates déjà les premiers mots indiquent que le Christ sauve : Il libère et transmet la grâce. Autrement dit le Christ unit, donne la divine plénitude de la vie ${ }^{1}$. «Paul, apôtre, non de la part des hommes, ni par un homme, mais

En ce qui concerne la signification du terme grâce cf. par ex. J. Guillet, Grâce, in: Vocabulaire de théologie biblique. Publié sous la direction de X. Léon-Dufour etc. Deuxième 
par Jésus Christ et Dieu le Père qui l'a ressuscité d'entre les morts, et tous les frères qui sont avec moi, aux Églises de Galatie: à vous grâce et paix de la part de Dieu notre Père et du Seigneur Jésus Christ, qui s'est livré pour nos péchés, afin de nous arracher à ce monde du mal, conformément à la volonté de Dieu, qui est notre Père. À lui soit la gloire pour les siècles des siècles. Amen» (Ga 1,1-5)². Dans quelques passages de l'Épître aux Galates l'Apôtre présente ce mystère comme la vie dans le Christ ${ }^{3}$. Aujourd'hui, quand si souvent on évoque la théorie de la globalisation, ne serait-il pas utile de parler de cette conception et expérience chrétiennes de la communion riche en deux dimensions inséparables: verticale et horizontale? L'enseignement des papes Jean Paul II et Benoît XVI montre qu'il faut ici donner la réponse positive ${ }^{4}$.

\section{La bénédiction paternelle}

«Christ a payé pour nous libérer de la malédiction de la loi, en devenant lui-même malédiction pour nous [...]. Cela pour que la bénédiction d'Abraham parvienne aux païens en Jésus Christ, et qu'ainsi nous recevions, par la foi, l'Esprit, objet de la promesse» (Ga 3,13-14)5. L'Apôtre parle ici d'une bénédiction très importante qui appartient selon lui aux avènements ouvrants la nouvelle, meilleure étape de l'histoire de la création-rédemption ( $c f$. par ex. Ga 4,1-7). Ces avènements marquent le fort positif progrès dans l'accomplissement de l'œuvre du salut. Dieu a choisi Abraham qui devient par conséquent le père du peuple-dépositaire de la rédemption. Ce peuple obtient de la part de Dieu la

édition révisée et augmentée, Paris 1971, col. 512s; G. Kraus, Nauka o łasce, Podręcznik teologii dogmatycznej, éd. W. Beinert, t. IX, Kraków 1999, p. 53n.

2 Les citations bibliques seront empruntées à La Bible. Traduction oecuménique. TOB, ParisPierrefitté 1988 .

$3 C f$. M.A. Seifrid, In Christ, in: Dictionary of Paul and His Letters. Editors: G.F. Hawthorne etc., Downers Grove-Leicester 1993, p. 433s.

4 Cf. par exemple: Benoît XVI, Verbum Domini. Exhortation apostolique post-synodale aux évêques, au clergé, aux personnes consacrées et aux fidèles laïcs sur la parole de Dieu dans la vie et dans la mission de l'Eglise (30.09.2010), nr 113; Benoît XVI, Silence et Parole: chemin d'évangélisation. Message pour la 46-ème journée mondiale des communications sociales (24.01.2012); Benoît XVI, Visita pastorale a Lamezia Terme e a Serra san Bruno, vespri, omelia: Chiesa della Certosa di Serra San Bruno (9.10.2011); Jean Paul II, Le progrès rapide. Lettre apostolique aux responsables des communications sociales (24.01.2005), $\mathrm{nr} 4$.

5 En Ga 3,14 la tournure «en Jésus Christ» correspond au grec en Christô Iêsou. Plus exacte donc est la traduction: dans le Christ Jésus. 
promesse du prospère avenir (autrement dit: du salut). Dans un sens il bénéficie même de son accomplissement ${ }^{6}$ : aussi pour les autres peuples, pour tous les hommes ( $c f$. par ex. Ga 3,6s). En conséquence nous avons à faire avec la bénédiction paternelle, donnée au père Abraham, donnée par le père Abraham. Mais pour st Paul cette bénédiction summa summarum provient de Dieu. C'est Dieu le Père bénit de cette manière ( $c f$. Ga $3,6 \mathrm{~s} ; 1,1 \mathrm{~s}$ ). Le caractère paternel de la bénédiction dont l'Apôtre parle en Ga 3,13-14, son caractère vital est donc renforcé de façon considérable puisque divine.

Un peu plus loin st Paul écrit : «tous, vous n'êtes qu'un en Jésus Christ» (Ga $3,28)^{7}$. On pourrait paraphraser ce dernier verset en disant que dans le Christ, que grâce à Lui aussi toute l'histoire redevient une et commune à tous comme efficace divine proposition du salut. D'un certain point de vu dans son stade entre Abraham et le Christ il y a le peuple élu et les nations païennes. Pour la compréhension de la situation de l'homme, de son sort futur cette division reste selon st Paul fort lourde de conséquence. «Nous sommes, nous, des Juifs de naissance et non pas des païens, ces pécheurs» $(\mathrm{Ga} 2,15)$. «[...] la colère de Dieu se révèle du haut du ciel contre toute impiété et toute injustice des hommes, qui retiennent la vérité captive de l'injustice» (Rm 1,18; cf. 1, 1932). On pourrait parler peut-être d'une certaine non-participation à l'œuvre du salut: «souvenez-vous qu'en ce temps-là, vous étiez sans Messie, privés du droit de cité en Israël, étrangers aux alliances de la promesse, sans espérance et sans Dieu dans le monde» (Eph 2,12) ${ }^{8}$. En tout cas sûrement l'état de nonparticipation ne jouit pas du privilège d'être insurmontable. Des l'avènement $\mathrm{du}$ Christ les païens seront pleinement intégrés (ou: réintégrés) à la communauté divine ( $c f$. par ex. Ga 3,28-29 et 5,6). Grâce à Lui ils sont même

6 Quant à la question du degré de cet accomplissement, les lettres paulinnienes invitent à le considérer dans la perspective de la fidélité, et par conséquent de la justice, de la miséricorde, de la sagesse de Dieu, de la réalisation mystérieuse et efficace de son plan (cf. par ex. Rm 3,22s; 11,1s; 1 Co 2,1s; 2 Co 1,19s; Eph 1,3s).

7 Aussi en Ga 3,28 la tournure «en Jésus Christ» rend le grec en Christô Iêsou. Plus exacte donc est la traduction: dans le Christ Jésus.

8 Au lieu de «sans Messie» on peut traduire «sans Christ» car en grec il y a ici chôris Christou. La préposition chôris signifie aussi loin de (cf. A Greek-English Lexicon of the New Testament and Other Early Christian Literature. A Translation and Adaptation of the Fourth Revised and Augmented Edition of W. Bauer's Griechisch-Deutsches Wörterbuch zu den Schriften des Neuen Testaments und der übrigen urchristlichen Literatur by W.F. Arndt and F. Wilbur Gingrich. Second Edition Revised and Augmented by F. Wilbur Gingrich and F.W. Danker from W. Bauer's Fifth Edition, Chicago-London 1979, p. 890). Il s'agit donc de la situation contraire à celle présentée comme dans le Christ. 
devenus héritiers du patriarche, du père Abraham ( $c f$. Ga 3,29). Comme st Paul l'écrit en Ga 3,14 c'est dans le Christ aussi que la bénédiction d'Abraham parvient à eux. Ce message de l'Épitre aux Galates trouve un interlocuteur dans le chapitre onze de l'Épître aux Romains où st Paul prévoit une autre cardinale intégration. Ici nous ne citerons que Rm 11,26: «Et ainsi tout Israël sera sauvé, comme il est écrit: De Sion viendra le libérateur, il écartera de Jacob les impiétés». La même optique, la même économie de bénédiction on rencontre dans le chapitre deux de l' Épitre aux Éphésiens.

Le mot eulogia (fr. bénédiction) n'est employé dans la Lettre aux Galates qu'une seule fois: en $\mathrm{Ga} 3,14^{9}$. En Ga 3,8 et Ga 3,9 on rencontre respectivement encore les verbes eneulogeô (fr. bénir) et eulogeô (fr.: 1. dire du bien, louer, célébrer, rendre grâce; 2. bénir) ${ }^{10}$. Ce qu'ils décrivent, reste strictement lié avec la justification et il s'ensuit: «D'ailleurs l'Écriture, prévoyant que Dieu justifierait les païens par la foi, a annoncé d'avance à Abraham cette bonne nouvelle: Toutes les nations seront bénies en toi (en soi). Ainsi donc, ceux qui sont croyants sont bénis avec Abraham, le croyant» (Ga 3,8-9) ${ }^{11}$. Il y a une correspondance entre «Toutes les nations seront bénies en toi (en soi)» en Ga 3,8 et «la bénédiction d'Abraham parvienne aux païens en Jésus Christ (en Christô Iêsou)» en Ga 3,14, entre «en toi (en soi)» et «dans le Christ Jésus (en Christô Iêsou)». On peut comprendre Ga 3,14 de la manière suivante: c'est le Christ qui assure pleinement la réalisation des promesses, qui assure le bénit, prospère avenir ${ }^{12}$. En plus la conjonction hina lie ce dernier passage d'une manière étroite avec le verset 13: «le Christ nous a rachetés de la malédiction de la Loi» $(\mathrm{Ga} 3,13)^{13}$. Paradoxalement, après Abraham, aux promesses et à la

9 Au total dans le corpus paulinum on le trouve encore huit fois: en Rm 15,59; 16,18; 1 Co 10,16; 2 Co 9,5 (deux fois) et 9,6 (deux fois) ainsi qu'en Eph 1,3. Dans le Nouveau Testament on ne rencontre le mot eulogia que 16 fois. Il est donc relativement rare.

${ }^{10}$ M. Carrez, F. Morel, Dictionnaire grec-français du Nouveau Testament. $4^{e}$ édition revue et corrigée, Genève-Pierrefitte 1988, p. 91 et 107.

${ }^{11} C f$. Ga 3,6: «Puisque Abraham eut foi en Dieu et que cela lui fut compté comme justice».

${ }^{12}$ A propos du terme bénédiction cf. J. Guillet, Bénédiction, in: Vocabulaire de théologie biblique. Publié sous la direction de X. Léon-Dufour etc. Deuxième édition révisée et augmentée, Paris 1971, col. 120s.

${ }^{13}$ Si hêmas (fr. nous) ne désigne que la personne de l'Apôtre ou les chrétiens d'origine juive ( $c f$. Ga 2,15), il s'agirait des Juifs qui étaient sous le régime de la Loi et qui ne pouvaient satisfaire à ses charges ( $c f$. par ex. Ga 3,10 et Dt 27,26, cf. aussi ce que St Paul dit de lui-même en Ga 1,13s). Mais en Ga 3,13 le mot hêmas (fr. nous) peut désigner un groupe composé aussi bien des chrétiens d'origine juive que d'origine non-juive ( $c f$. Ga 1,2: «et tous les frères qui sont avec moi, aux Églises de Galatie»). Vu le contexte c'est cette dernière possibilité qui semble plus fondée ( $c f$. par ex. Ga 
bénédiction succède la malédiction ( $c f$. Ga 3,8-9 et 10-13) ${ }^{14}$. Ce n'est qu'avec le Christ, en Lui, qu'elle sera définitivement enlevée, que l'homme pourra être justifié. «[...] tous ont péché, sont privés de la gloire de Dieu, mais sont gratuitement justifiés par sa grâce, en vertu de la délivrance accomplie en Jésus Christ (en Christô Iêsou)» (Rm 3,23-24; cf. Ga 3,10.11.13.8-9).

La justification donnée par Dieu, c'est d'abord un acte, pour ainsi dire, juridique. Mais cet acte inaugure dans la vie de celui qui a été justifié, une nouvelle étape et un nouvel état dynamiques et vitaux qui sont ceux de justification-justice-sainteté et de bénédiction ${ }^{15}$. Ainsi ce qui était impossible pour tout homme sous le régime de la Loi, devient accessible à tous sous le régime nouveau, sous le Christ, en Lui:

\begin{tabular}{|l|l|}
\hline oudeis dikaioutai para tô theô en nomô (Ga 3,11) & $\begin{array}{l}\text { hina eis ta ethnê hê eulogia tou Abraam genêtai } \\
\text { en Christô Iêsou }(\mathrm{Ga} 3,14)^{\mathrm{I}}\end{array}$ \\
\hline $\begin{array}{l}\text { «Il est d'ailleurs évident que, par la loi, nul n’est } \\
\text { justifié devant Dieu, puisque celui qui est juste } \\
\text { par la foi vivra» (Ga 3,11) }\end{array}$ & $\begin{array}{l}\text { «Cela pour que la bénédiction d'Abraham } \\
\text { parvienne aux païens en Jésus Christ, et } \\
\text { qu'ainsi nous recevions, par la foi, l'Esprit, objet } \\
\text { de la promesse» (Ga 3,14) }\end{array}$ \\
\hline
\end{tabular}

I Il y a aussi la leçon en Iêsou Christô (fr. en Jésus Christ). Cf. l'apparat critique.

En citant respectivement $\mathrm{Ga} 3,10$ et 3,9 on pourrait prolonger ce tableau de façon suivante:

\begin{tabular}{|l|l|}
\hline $\begin{array}{l}\text { hosoi ex ergôn nomou eisin, hypo kataran eisin } \\
(\mathrm{Ga} 3,10)\end{array}$ & $\begin{array}{l}\text { hôste hoi ek pisteôs eulogountai syn tô pistô } \\
\text { Abraam }(\mathrm{Ga} 3,9)\end{array}$ \\
\hline $\begin{array}{l}\text { «Car les pratiquants de la loi sont tous sous } \\
\text { le coup de la malédiction, puisqu'il est écrit: } \\
\begin{array}{l}\text { Maudit soit quiconque ne persévère pas dans } \\
\text { l'accomplissement de tout ce qui est écrit dans le } \\
\text { livre de la loi» }(\mathrm{Ga} 3,10)\end{array}\end{array}$ & $\begin{array}{l}\text { «Ainsi donc, ceux qui sont croyants sont bénis } \\
\text { avec Abraham, le croyant» }(\mathrm{Ga} 3,9)\end{array}$ \\
\hline
\end{tabular}

3,7: «comprenez-le donc: ce sont les croyants qui sont fils d'Abraham»). Tous, ils ont besoin d'être justifiés.

${ }^{14}$ Il faut préciser «aussi la malédiction» car la malédiction n'est pas seule et avant tout elle n'est pas le plus important, décisif élément.

${ }^{15}$ R. Penna explique: «La classica interpretazione luterana, che riconosce il cristiano 'giusto e peccatore insieme', lascia le cose solo a metà e di fatto ritiene Dio incapace ad operare quella che invece, per S. Paulo, è addirittura una nuova creazione: 'se qualcuno è in Cristo, è una nuova creatura; le cose vecchie sono passate: tutto è diventato nuovo' ( 2 Cor 5,17$)$. La cosa incomparabile non è solo che Dio-giudice dica al malvagio: 'Tu sei innocente'; ma pure che lo crea tale. Tutt'altro che considerare il cristiano 'come se' fosse giusto, Paulo è cosciente che la grazia di Dio trasforma ciò che tocca» (Essere cristiani secondo Paolo, Casale Monferrato 1979, p. 48). 
Pour st Paul, l'homme, qu'il soit d'origine juive ou païenne, n'est pas justifié par (gr. $e k$ ) les oeuvres de la Loi, mais par (gr. $e k$ ) la foi ( $c f$. Ga 3,8.10-11; 2,16 et 3,8$)^{16}$. D'après l'Apôtre par (gr. $e k$ ) la foi aussi et indépendamment de ses origines juives ou non juives, chaque chrétien est fils d'Abraham, héritier de l'alliance et des promesses que Dieu a faites à ce patriarche: «comprenez-le donc: ce sont les croyants qui sont fils d'Abraham (hoi ek pisteôs, houtoi huioi eisin Abraam)» (Ga 3,7). De même la vie nouvelle qui est propre aux chrétiens découle-t-elle dans une certaine mesure de cette partie de l'histoire du salut qui s'identifie avec l'histoire du peuple élue d'Abraham jusqu'au Christ. Son héritage et ses bénédictions deviennent les siens ( $c f$. Rm 11,1s) ${ }^{17}$.

Le Christ sauve des conséquences de l'inobservance de la Loi ( $c f$. Ga 3,10.12), c'est-à-dire de sa malédiction: «Christ a payé pour nous libérer de la malédiction de la loi, en devenant lui-même malédiction pour nous, puisqu'il est écrit: Maudit quiconque est pendu au bois» $(\mathrm{Ga} 3,13)^{18}$. C'est en Lui que tous sont justifiés ( $c f$. Ga 2,16s). La justification annonce et prépare un changement définitif, eschatologique et, en même temps, elle signifie le début de ce changement dans la vie de l'homme ( $c f$. par ex. Col 2,12s). Comme telle elle ne lui vient que de Dieu et s'avère indispensable pour que

${ }^{16}$ Quant aux païens (gr. ta ethnê), d'après Ga 3,8 ce dessein de Dieu a déjà été révélé à Abraham: «D'ailleurs l'Écriture, prévoyant que Dieu justifierait les païens par la foi, a annoncé d'avance à Abraham cette bonne nouvelle: Toutes les nations seront bénies en toi» $(\mathrm{Ga} 3,8)$.

${ }^{17}$ Cf. H.W. Beyer, eulogeô etc., in: Theologisches Wörterbuch zum Neunen Testament, t. II, p. 761 et J. Jeremias, Abraam, in: Theologisches Wörterbuch zum Neunen Testament, t. I, p. 8.

${ }^{18}$ En Ga 3,6-14 st Paul parle de la situation des gens désignés respectivement par hoi ek pisteôs (3,7.9: «ce sont les croyants qui sont fils d'Abraham ... ceux qui sont croyants sont bénis avec Abraham, le croyant»), ta ethnê (3,8.14: «D'ailleurs l'Écriture, prévoyant que Dieu justifierait les païens par la foi, a annoncé d'avance à Abraham cette bonne nouvelle: Toutes les nations seront bénies en toi ... Cela pour que la bénédiction d'Abraham parvienne aux païens en Jésus Christ, et qu'ainsi nous recevions, par la foi, l'Esprit, objet de la promesse»), hosoi ex ergôn nomou (3,10: «Car les pratiquants de la loi sont tous sous le coup de la malédiction, puisqu'il est écrit: Maudit soit quiconque ne persévère pas dans l'accomplissement de tout ce qui est écrit dans le livre de la loi»), hêmeis (3,13ab: «Christ a payé pour nous libérer de la malédiction de la loi, en devenant luimême malédiction pour nous, puisqu'il est écrit: Maudit quiconque est pendu au bois») et labômen (3,14: «Cela pour que la bénédiction d'Abraham parvienne aux païens en Jésus Christ, et qu'ainsi nous recevions, par la foi, l'Esprit, objet de la promesse»). L'appartenance à un de ces groupes n'exclut pas à priori l'appartenance à un autre. $C f$. par ex. le jeu entre ta ethnê et hêmeis en Ga 3,8.13 qui ne s'identifient pas tout simplement. Les appartenances à ces groupes sont plutôt assez souples. «D’ailleurs l'Écriture, prévoyant que Dieu justifierait les païens (ta ethnê) par la foi, a annoncé d'avance à Abraham cette bonne nouvelle: Toutes les nations (ta ethnê) seront bénies en toi ... Christ a payé pour nous (hêmas) libérer de la malédiction de la loi, en devenant lui-même malédiction pour nous (hêmôn), puisqu'il est écrit: Maudit quiconque est pendu au bois» (Ga 3,8.13). 
l'homme renaisse. Étant ici condicio sine qua non elle se place au seuil de la vie nouvelle. "Aussi, si quelqu'un est en Christ, il est une nouvelle créature. Le monde ancien est passé, voici qu'une réalité nouvelle est là» (2 Co 5,17). «Car, ce qui importe, ce n'est ni la circoncision, ni l'incirconcision, mais la nouvelle création» $(\mathrm{Ga} 6,15)$. La justification est un des plus grands dons divins et la Parole de Dieu ira jusqu'à l'identifier avec une des promesses divines faites à Abraham $(\mathrm{Ga} 3,8-9)^{19}$. Celle-ci se réalisera ( $c f$. genêtai en $\mathrm{Ga} 3,14)$, aussi pour les païens, dans le Christ dès son mystère pascal (Ga 3,13-14; cf. 1 Co 15,15s $)^{20}$. St Paul est conscient et veut que les autres sachent qu'il est témoin de ce processus décisif pour la bonne réussite de la vie de l'homme et de l'histoire du monde, qu'ils y participent ensemble avec lui ( $c f$. par ex. Rm 9,1s et 1 Co 9,19s).

\section{Le Fils et les fils}

D'après l'Épître aux Galates le cœur de l'œuvre de la création-rédemption est aussi constitué par le mystère connu sous le nom adoption. «Et nous, de même, quand nous étions des enfants soumis aux éléments du monde, nous étions esclaves. Mais, quand est venu l'accomplissement du temps, Dieu a envoyé son Fils, né d'une femme et assujetti à la loi, pour payer la libération de ceux qui sont assujettis à la loi, pour qu'il nous soit donné d'être fils adoptifs. Fils, vous l'êtes bien: Dieu a envoyé dans nos cœurs l'Esprit de son Fils, qui crie: Abba - Père! Tu n'es donc plus esclave, mais fils; et, comme fils, tu es aussi héritier: c'est l'œuvre de Dieu» (Ga 4,3-7; cf. Rm 8,11-17; Eph 1,3-14; 2,1-19). Le Catéchisme de l'Église Catholique explique cette économie de manière suivante: Dieu «convoque tous les hommes que le péché a dispersés dans l'unité de sa famille, l'Église. Pour ce faire, Il a envoyé son Fils comme Rédempteur et Sauveur lorsque les temps furent accomplis. En Lui et par Lui, Il appelle les hommes à devenir, dans l'Esprit Saint, ses enfants d'adoption, et

${ }^{19}$ F. Zorell (Lexicon graecum Novi Testamenti. Editio tertia quae est editio altera. Iterum typis mandata. Appendice bibliographica adiecta, Parisiis 1961, p. 534) traduit eulogia tou Abraam de Ga 3,14 par «bona Abrahamo promissa» (cf. Ga 3,8-9).

${ }^{20}$ Cf. st Léon le Grand, Sermon XII sur la passion, 6: «Ipse est in quo semen Abrahae totius mundi adoptione benedicitur; et fit patriarcha gentium pater, dum promissionis filii fide, non carne nascuntur» (Léon le Grand, Sermons, t. III, Sources Chrétiennes 74, Paris 1961, p. 82). 
donc les héritiers de sa vie bienheureuse» ${ }^{21}$. Dieu offre, propose ce don à tous. «Je serai pour vous un père, et vous serez pour moi des fils et des filles, dit le Seigneur tout-puissant» $(2 \text { Co } 6,18)^{22}$. On trouve la même idée dans la Lettre aux Galates: «Car tous, vous êtes, par la foi, fils de Dieu, en Jésus Christ. [...] Il n'y a plus ni Juif, ni Grec; il n'y a plus ni esclave, ni homme libre; il n'y a plus l'homme et la femme; car tous, vous n'êtes qu'un en Jésus Christ» $(\mathrm{Ga} 3,26.28)^{23}$.

Comme l'indique la Lettre aux Galates, les chrétiens sont fils de Dieu dia tês pisteôs en Christô Iêsou: «tous, vous êtes, par la foi, fils de Dieu, en Jésus Christ (en Christô Iêsou)» (Ga 3,26). La question qui se pose immédiatement, c'est le problème de l'interprétation de la construction pistis $+e n$. On rencontre les constructions du même type en Eph 1,15: «j'ai appris votre foi dans le Seigneur Jésus (tên kath'hymas pistin en tô kyriô Iêsou)»; Col 1,4: «nous avons entendu parler de votre foi en Jésus Christ (akousantes ten pistin hymôn en Christô Iêsou)»; $1 \mathrm{Tm}$ 3,13: «ceux qui exercent bien le ministère de diacre s'acquièrent un beau rang ainsi qu'une grande assurance fondée sur la foi qui est dans le Christ Jésus (en pistei tê en Christô Iêsou)»; 2 Tm 3,15: «Depuis ta tendre enfance tu connais les Saintes Écritures; elles ont le pouvoir de te communiquer la sagesse qui conduit au salut par la foi qui est dans le Christ Jésus (dia pisteôs tês en Christô lêsou)» et, à la limite, en Rm 3,25: «C'est lui que Dieu a destiné à servir d'expiation par son sang, par le moyen de la foi (dia tês pisteôs en tô autou haimati) $\rangle^{24}$.

${ }^{21}$ Catéchisme de l'Église Catholique, Paris 1992, p. 11, § 1. Les mots cités se trouvent juste au début du Catéchisme. Ils ouvrent son message sur le projet de Dieu ainsi que sur son accomplissement. Cf. aussi C. Kourie, Understanding Paul - The Insights of Dom Columba Mariom and Elisabeth of the Trinity, in: Studies in Spirituality 14 (2004), p. 37 s.

${ }^{22}$ En 2 Co 6,18 st Paul reprend le message de l'Ancien Testament (cf. Is 4,36; 2 S 7,8; Am $3,13)$. Nous avons donc à faire avec une importante, colorée d'optimisme, continuité théologiquespirituelle.

${ }^{23}$ Il s'agit ainsi du statut des femmes. P.-D. Dognin explique: «Il n'y a ni homme ni femme [...]. Cette affirmation peut être mise en rapport avec le fait que les femmes sont baptisées aussi bien que les hommes. Ce contraste entre le baptême et la circoncision avait été déjà noté par saint Justin» ("La foi étant venue». l'Épître aux Galates, Bruxelles 2001, p. 48). Il s'agit d'une capitale correction en faveur des femmes de leur position telle que le monde greco-romain la considère. $C f$. C.S. Keener, Man and Woman, in: Dictionary of Paul and His Letters. Editors: G.F. Hawthorne etc., Downers Grove-Leicester 1993, p. 583s.

${ }^{24}$ En ce qui concerne Ga 3,26 il y a aussi les leçons dia pisteôs Christou lêsou, dia pisteôs Iêsou Christou et dia pisteôs, cf. l'apparat critique. La Vulgate traduit le verset en question par: «omnes enim filii Dei estis per fidem in Christo Iesu» (Biblia Sacra. Iuxta vulgatam versionem, Stuttgart 1983, cf. l'apparat critique), Die Bibel. Einheitsübersetzung der Heiligen Schrift. 
Si en Ga 3,26la formulation en Christô Iêsou (fr. dans le Christ) ne dépend pas de dia tês pisteôs (fr. par la foi ${ }^{25}$, la formule en question servirait à indiquer non seulement une certaine relation entre le Christ et les chrétiens (par ex. celle d'une appartenance à Lui ou d'une union avec Lui, cf. Ga 3,27 et 3,29), mais aussi ce que cette relation produit, ce qu'elle signifie pour le croyant: les hommes sont fils de Dieu dans le Christ. Ils le deviennent en Lui, grâce à Lui (ce que st Paul dit explicitement en Ga 4,4-7). D'un certain point de vue cette relation se base sur la foi et le baptême: elle est, elle commence et s'approfondit jusqu'à la plénitude eschatologique par la foi et par le baptême. La foi est mentionnée dans le même Ga 3,26. Du baptême l'Apôtre parle dans le verset suivant: «Oui, vous tous qui avez été baptisés en Christ (eis Christôn), vous avez revêtu Christ» (Ga 3,27). Au cas où en Ga 3,26 la formulation en Christô lêsou devrait être considérée comme un complément de dia tês pisteôs, il s'agirait d'une explicite indication de la foi en Christ comme celle qui se trouve à la «base» de l'adoption divine ${ }^{26}$. On peut par conséquent identifier un des traits les plus fondamentaux de la foi chrétienne: il s'agit de la relation de caractère paternel-filial. La foi en Christ qui fait de l'homme le fils de Dieu, est d'après les lettres pauliniennes en même temps don divin et réponse de l'homme (cf. par ex 1 Co 12,3s).

Le concept d'adoption (gr. huiothesia) dont parle Ga 3,26 et Ga 4,3-7, résume d'une certaine façon toute l'économie du salut. Bien entendu ce don

Gesamtausgabe (Stuttgart - Klosterneuburg 1987) par: «Ihr seid alle durchden Glauben Söhne Gottes in Christus Jesus», et Parola del Signore. Il Nuovo Testamento in lingua corrente (Torino - Roma 1988) par: «Voi tutti siete figli di Dio per mezzo di Gesù Cristo, perché credete in lui». Cf. R.E. Brown, Que sait-on du Nouveau Testament? Présentation de P. Debergé, Paris 2000, p. 522s.

${ }^{25}$ Cf. J.A. Fitzmyer, The Letter to the Galatians, in: The New Jerome Biblical Commentary, éd. R.E. Brown S.S. etc., London 1992, p. 786; F. Zorell, Lexicon Graecum Novi Testamenti. Editio tertia quae est editio altera. Iterum typis mandata. Appendice bibliographica adiecta, Parisiis 1961, col. 1064.

${ }^{26}$ En Ga 3,26 on pourrait donc comprendre les mots dia tês pisteôs en Christô Iêsou dans le sens de dia tês pisteôs eis Christon. Cf. R. Bultmann, pisteuô. D. Die Begriffsgruppe pistis im NT, in: R. Bultmann, A. Weiser, pisteuô etc., in: Theologisches Wörterbuch zum Neuen Testament, t. VI, p. 204; A. Oepke, eis, in: Theologisches Wörterbuch zum Neuen Testament, t. II, p. 431s; W. Marchel, Abba. Père. La prière du Christ et des chrétiens. Etudes sur les origines et la signification de l'invocation à la divinité comme père avant et dans le Nouveau Testament, Rome 1971, p. 208, A. Descamps, Le baptême, fondement de l'unité chrétienne, in: Battesimo e giustizia in Rom 6 e 8. A cura di L. De Lorenzi etc. Roma 1974, p. 228s; F. Zorell, Lexicon Graecum Novi Testamenti, Editio tertia quae est editio altera. Iterum typis mandata. Appendice bibliographica adiecta, Parisiis 1961, col. 1064. 
ne s'accomplit que grâce au Christ, par Lui, en Lui, dans la communion avec Lui. Cela signifie aussi, comme par exemple Ga 4,3-7 indique, l'engagement efficace de la par de Dieu le Père et de l'Esprit Saint et l'étroite communion aussi avec Eux ( $c f$. aussi par ex. Rm 8,14-17). En Ga 3,26 st Paul met l'accent sur le rôle du Christ dans ce mystère. Et c'est dans ce sens qu'on peut aussi interpréter la formule dans le Christ Jésus dans ce texte.

\section{Tous, vous n'êtes qu'un dans le Christ Jésus}

Tous ceux qui sont devenus, comme chrétiens, fils de Dieu (Ga 3,26), qui ont été baptisés dans le Christ (gr. eis Christon, Ga 3,27) et qui L'ont ainsi revêtu (Ga 3,27), ils ne sont qu'un en Christ Jésus. «Il n'y a plus ni Juif, ni Grec; il n'y a plus ni esclave, ni homme libre; il n'y a plus l'homme et la femme; car tous, vous n'êtes qu'un en Jésus Christ (pantes gar hymeis heis este en Christô Iêsou)» (Ga 3,28) ${ }^{27}$. En fin de compte pour eux toutes les divisions, même celles les plus fondamentales, sont donc, dans le Christ, comme inexistantes ou périmées. Surtout elles ont été révisées, réévaluées et ne nuisent plus (au moins les chrétiens sont en état de bénéficier de cette nouvelle situation). Dans le même sens conduit Col 3,9-11: «[...] vous vous êtes dépouillés du vieil homme, avec ses pratiques, et vous avez revêtu l'homme nouveau, celui qui, pour accéder à la connaissance, ne cesse d'être renouvelé à l'image de son créateur; là, il n'y a plus Grec et Juif, circoncis et incirconcis, barbare, Scythe, esclave, homme libre, mais Christ: il est tout et en tous $\rangle^{28}$.

On peut lire dans les mots «vous n'êtes qu'un en Jésus Christ (en Christô Iêsou)» (Ga 3,28) une idée d'appartenance des chrétiens au Christ par ex. au sens de l'incorporation au Corps mystique du Christ ${ }^{29}$. En effet dans la

${ }^{27} \mathrm{Au}$ lieu de pantes quelques manuscrits ont hapantes (fr. tous ensemble). Il y a aussi les leçons hen este en Christô, este Christou, este hen Christou. Cf. l'apparat critique.

${ }^{28}$ Il sera utile d'invoquer ici l'inscription du pape Sixte III (432-440) du baptistère du Latran à Rome: «[...] NVLLA RENASCENTVM EST DISTANTIA QVOS FACIT VNVM VNVS FONS VNVS SPIRITVS VNA FIDES [...]» (cité d'après H. Leclerque, Latran, in: Dictionnaire d'archéologie chrétienne et de liturgie. Tome huitième. Deuxième partie, Paris 1907, col. 1558).

${ }^{29}$ Cf. J.A. Fitzmyer, The Letter to the Galatians, in: The New Jerome Biblical Commentary, éd. R.E. Brown S.S. etc., London 1992, p. 787; B. Corsani, Lettera ai Galati, Genova 1990, p. 383; R. Bultmann, Theologie des Neuen Testaments, Tübingen 1980, p. 312 («Das en Christô ... ist primer eine ekklesiologische Formel und bezeichnet das Eingefügtsein in das sôma Christou durch die Taufe, wenn auch nicht in jedem Falle ausdrücklich an die Taufe gedacht zu sein braucht ..., und 
Lettre aux Romains st Paul explique: «ainsi, à plusieurs, nous sommes un seul corps en Christ (en Christô), étant tous membres les uns des autres, chacun pour sa part» (Rm 12,5). En Ga 3,27 il est question de l'union avec le Christ et plus précisément d'une incorporation baptismale des chrétiens à Lui. Un message fort semblable nous rencontrons dans la Première lettre aux Corinthiens: "Car nous avons tous été baptisés dans un seul Esprit (en heni pneumati) en (eis) un seul corps, Juifs ou Grecs, esclaves ou hommes libres» (1 Co 12,13). Selon st Paul l'appartenance au Christ est bénéfique pour l'homme. Il s'agit d'un état qui ouvre devant les hommes la participation aux promesses et à l'œuvre divine ainsi qu'à leur plein accomplissement eschatologique. «Et si vous appartenez au Christ, c'est donc que vous êtes la descendance d'Abraham; selon la promesse, vous êtes héritiers» (Ga 3,29). «Cet Esprit lui-même atteste à notre esprit que nous sommes enfants de Dieu. Enfants, et donc héritiers: héritiers de Dieu, cohéritiers du Christ, puisque, ayant part à ses souffrances, nous aurons part aussi à sa gloire» (Rom 8,1617). L'appartenance au Christ, la soumission, l'obéissance à Lui demeure pour l'homme un statut entièrement positif. Elle s'identifie par exemple avec la libération, avec la liberté. «C'est pour que nous soyons vraiment libres que Christ nous a libérés. Tenez donc ferme et ne vous laissez pas remettre sous le joug de l'esclavage» (Ga 5,1; cf. 4,1-7; 5,13). En commentant 2 Co 1,22; 5,5; $\mathrm{Rm} 8,23$ et Ga 5,17 un théologien explique: «C'est pourquoi, il n'y a aucune illusion à avoir: la liberté qu'est la nôtre n'est pas autonomie. Dans le désir d'autonomie s'exprime notre prétention trompeuse à la suffisance éthique, comme Adam souhaitant s'approprier la connaissance du bien et du mal. Ce qui nous est donné 'en Christ', c'est précisément [...] la possibilité d'échapper à l'illusion de l'autonomie, la possibilité de voir clairement que le choix est entre les pesanteurs diverses de la chaire et la conduite par l'Esprit de Dieu, par le souffle de Dieu. Paul n'hésite pas à dire: notre liberté, c'est d'obéir a la loi (!) du souffle, au lieu d'obéir à la loi du péché et de la chair $(\mathrm{Rm} 8 / 2$ et la suite) $)\rangle^{30}$. Mutatis mutandis ces remarques s'appliquent bien aussi aux relations chrétiens - Christ (cf. par ex. Rm 8,9s; Ga 6,1s).

wenn auch die Formel da aus eine erweiterte Gebrauch gewonnen hat und oft nur allgemein die Bestimmtheit durch Christus ausdrückt und ein noch fehlendes Adjektive oder Adverb ,christlich ersetzt»).

${ }^{30}$ M.-A. Chevallier, Souffle de Dieu. Le Saint-Esprit dans le Nouveau Testament, t. III Etudes. Avant-propos de J.-N. Aletti, Paris 1991, p. 152s. 


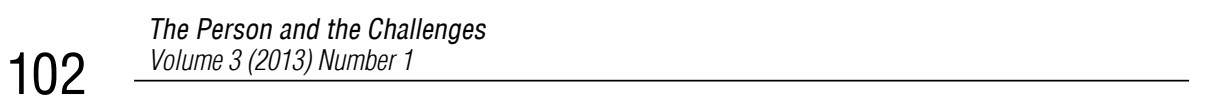

En même temps Ga 3,28 semble mettre l'accent sur la communion entre les hommes. Si le péché divise et oppose aussi les hommes entre eux ( $c f$. par ex. Ga 5,14s), la communion avec Christ porte la victoire pareillement sur cette conséquence du péché. On peut comprendre la formule en Christô Iêsou en Ga 3,28 comme une sorte d'indication que l'unité dont il est question, ne se réalise que dans le Christ, grâce à Lui. D'après la Lettre aux Éphésiens nous avons ici à faire avec une unification du caractère universelle, cosmique. «Il [Dieu le Père] nous a fait connaître le mystère de sa volonté, le dessein bienveillant qu'il a d'avance arrêté en lui-même pour mener les temps à leur accomplissement: réunir l'univers entier sous un seul chef, le Christ (en tô Christô), ce qui est dans les cieux et ce qui est sur la terre» (Eph 1,9-10; $c f$. Rm 8,18-23) ${ }^{31}$. Le nouveau statut de l'homme (et du monde) l' $^{32}$ acquis dans le Christ est tel qu'il prévaut donc contre l'ordre ancien et ses nuisibles divisions ( $c f .2$ Co 5,17) $)^{33}$. Il fait des hommes qui en bénéficient, la descendance d'Abraham et les fils de Dieu. Désormais tous doivent comprendre qu'ils sont égaux. Tous ne sont qu'un dans le Christ Jésus ( $c f$. Ga 2,20). Cela ne signifie pas une perte de l'individualité ou un renoncement à l'identité propre de la personne ${ }^{34}$ mais un développent, un progrès spirituels ( $c f$. par ex. Ga 6,1s; Rm 12,1s; 1 Co 12,1s).

\section{Le renouvellement et l'accomplissement de la création}

Selon st Paul le Christ unit dans l'histoire de la création-rédemption. Il fait participer ceux qui ont besoin du salut dans la richesse de ses bénéfices. «Car, pour celui qui est en Jésus Christ (en gar Christô Iêsou), ni la circoncision, ni l'incirconcision ne sont efficaces, mais la foi agissant par l'amour» $(\mathrm{Ga} 5,6)$.

${ }^{31}$ Cf. T. Preiss, La vie en Christ. Préface de R. Mehl, Neuchâtel-Paris 1951, p. 98, W. Marchel, Abba. Père. La prière du Christ et des chrétiens. Etudes sur les origines et la signification de l'invocation à la divinité comme père avant et dans le Nouveau Testament, Rome 1971, p. 209s.

32 J.B. Green remarque: «Finally, it is remarkable that, for Paul, sin marks a rupture in the divine-human relations and in relations between humanity and the material creation» (Death of Christ, in: Dictionary of Paul and His Letters. Editors: G.F. Hawthorne etc., Downers GroveLeicester 1993, p. 207).

${ }^{33}$ Cf. J. Behm, kainos etc., in: Theologisches Wörterbuch zum Neuen Testament, t. III, p. 450.

${ }^{34} \mathrm{Cf}$. F. Vouga, La vérité de l'Évangile et la création nouvelle: l'apôtre Paul comme interprète de Jésus de Nazareth, in: Paul, une théologie en construction. Sous la direction de A. Dettwiler etc., Genève 2004, p. 359; W. Misztal, Duchowość chrześcijan w świetle listów Pawłowych: zwiazki z Bogiem, Kraków 2010, p. 61s; 240s. 
L'Apôtre est soucieux d'en donner les explications afin que la personne donnée puisse en tirer les avantages.

Personnellement st Paul a dû affronter aussi le cas suivant. La rencontre entre les chrétiens provenant du judaïsme et ceux venant du paganisme devait poser la question du statut de l'héritage de l'Ancienne Alliance, par ex. de ses prescriptions ${ }^{35}$. Parmi ces dernières il faut peut-être citer tout d'abord la circoncision $^{36}$. Elle signifiait l'adhésion au peuple d'Israël et à son alliance avec toutes les conséquences qui en dérivaient. Finalement ce mot désignera même l'ensemble de tous ceux qui étaient censés observer la Loi mosaïque ( $c f$. par ex. Ga 2,8).

Ainsi d'un certain point de vue l'humanité entière se divisait-elle en circoncis (gr. peritomê) et incirconcis (gr. akrobystia; cf. par ex. Ga 2,7.9). On pourrait donc dire qu'il y a deux groupes, deux ordres antagonistes. Les chrétiens se recrutent dans le premier et dans le second groupe. Et le premier et le second ordre cèdent la place à la nouvelle économie du salut, à l'ordre nouvel instauré par le Christ. C'est dans ce sens qu'on peut comprendre la formule dans le Christ Jésus (gr. en ... Christô Iêsou) ${ }^{37}$ en Ga 5,6. En Ga 5,4 st Paul explique, avertit: «Vous avez rompu avec Christ, si vous placez votre justice dans la loi (en nomô); vous êtes déchus de la grâce». A la lumière de ce dernier texte il y a alors un choix entre deux états. Mais absolument il n'est pas permit d'oublier le caractère polémique et non pas systématique qui est propre au raisonnement de st Paul dans la Lettre aux Galates $^{38}$ : cette épître n'est pas le dernier mot de l'Apôtre, elle ne présente pas sa pensée d'une façon exhaustive. D'autres textes pauliniens obligent d'envisager le suivant status $q u o$. Les hommes qui ont tous besoin du salut provenant de Dieu, peuvent se trouver dans un de ces trois états: sans Christ (il s'agit des païens; $c f$. par ex. Eph 2,12), sous la Loi (la synagogue; cf. par ex. Ga 3,23) et dans le Christ (les

${ }^{35}$ Cf. par ex. U. Vanni, Duchowość Pawła, in: Historia duchowości. Tom II. Duchowość Nowego Testamentu, Kraków 2003, p. 148s.

${ }^{36}$ Cf. par ex. Obrzezanie, in: Stownik symboliki biblijnej. Obrazy, symbole, motywy, metafory, figury stylistyczne i gatunki literackie w Piśmie Świętym. Ed. L. Ryken, J.C. Wilhoit, T. Longman, Warszawa 2003, p. 616s; S. Légasse, Paul apôtre. Essai de biographie critique. Deuxième édition revue, Paris 2000 , p. $107 \mathrm{~s}$ et $143 \mathrm{~s}$.

${ }^{37}$ Il y a aussi la leçon en ... Christô (fr. dans le Christ). Cf. l'apparat critique.

${ }^{38}$ Cf. S. Légasse, Paul apôtre. Essai de biographie critique. Deuxième édition revue, Paris 2000 , p. 155s; A. Pitta, Lettera ai Galati. Introduzione, versione, commento, Bologna 1996, p. 15s; R. Pindel, W obronie prawdy Ewangelii. Analiza literacko-historyczna Ga 1,11 - 2,21, Kraków 2001, p. 21s. 
chrétiens; $c f$. par ex. Ga 5,6). Par leur nature ces états sont d'ordre religieux et non pas ethnique, national ou chronologique. La personne donnée a la possibilité de passer de l'un à l'autre de ces états. Entre l'étape sous la Loi et l'étape dans le Christ il a une importante, très spécifique continuité dont st Paul parle largement par ex. en Rm 9 - 1139. Étant donné que le Christ joue un rôle fondamental dans l'œuvre du salut ( $c f$. par ex. Ga 2,17s) l'état (l'étape) dans le Christ se révèle comme le plus important, le plus avantageux, comme le but ( $c f$. par ex. 1 Co 15,22s). Mais il faut aussi souligner une notable continuité entre le premier état-stade et le second état-stade ( $c f$. Ga 3,6s ; Rm $4,9 \mathrm{n})^{40}$ ainsi qu'entre le premier état-stade et le troisième état-stade ( $c f$. Col $1,15 \mathrm{~s})$. Cela garantie que l'histoire du monde créé reste l'histoire du salut. Si l'état-stade dans le Christ est le but de cette histoire ( $c f$. par ex Rm 6,23 et 1 Co 15,21s) en même temps d'après les lettres de st Paul les relations avec le Christ, son aide efficace et donc aussi une certaine salvatrice communion avec Lui modèlent déjà le premier état-stade et le second état-stade ( $c f .1 \mathrm{Co}$ $10,1 \mathrm{~s} ; \mathrm{Col} 1,15 \mathrm{~s}$ et Ef 2,10$)^{41}$.

La nouvelle économie du salut, l'économie dans le Christ est celle de la foi: sûrement pas de la foi passive, mais celle qui agit, transforme positivement par amour (gr. agapê) ${ }^{42}$. On pourrait citer ici par ex. Ga 5,5; 2,16; 3,8.14.2226; 2,20 ainsi que 5,13.22 et 1 Co 12,31s. L'amour vient d'abord de Dieu qui le transmet: «je vis, mais ce n'est plus moi, c'est Christ qui vit en moi. Car ma vie présente dans la chair, je la vis dans la foi au Fils de Dieu qui m'a aimé et s'est livré pour moi» (Ga 2,20). Une fois cette condition accomplie, l'amour vient de l'homme, il devient le sien comme une réponse possible et attendue. «Vous, frères, c'est à la liberté que vous avez été appelés. Seulement, que cette liberté ne donne aucune prise à la chair! Mais, par l'amour, mettez-vous au service les uns des autres. Car la loi tout entière trouve son accomplissement en cette unique parole: Tu aimeras ton prochain comme toi-même» (Ga 5,13-

${ }^{39}$ Cf. par ex. F.X. Durrwell, Ojciec. Bóg w swoim misterium, Kielce 2000, p. 56n.

${ }^{40}$ Cf. Cothenet E., L'épître aux Galates, in : Cahiers Evangile 34 (1980), p. 36s.

${ }^{41} C f$. Gaudium et spes 22: «En effet, puisque le Christ est mort pour tous et que la vocation dernière de l'homme est réellement unique, à savoir divine, nous devons tenir que l'Esprit-Saint offre à tous, d'une façon que Dieu connaît, la possibilité d'être associé au mystère Pascal»; le texte emprunté à: www.vatican.va; le site visité: 9.02.2007; Lumen gentium 16; Catéchisme de l'Église Catholique 1260, Jean Paul II, Audience générale du 24.11.2004 «Le Seigneur Jésus 'Premier-Né' de toute créature et 'Premier-Né' d'entre les morts (lecture: Col 1,3.12.15-17)».

${ }^{42}$ Cf. G. Bertram, ergon etc., in: Theologisches Wörterbuch zum Neuen Testament, t. II, p. 651 et 646 . 
14). Cet amour unit étroitement, d'une manière intime Dieu et l'homme ainsi que les hommes entre eux-mêmes: «voici le fruit de l'Esprit: amour, joie, paix, patience, bonté, bienveillance, foi, douceur, maîtrise de soi» (Ga 5,22-23). Quant à la foi dont l'Apôtre parle en Ga 5,6, elle est la même pour les chrétiens d'origine juive que pour les chrétiens d'origine païenne. Dans le Christ ils sont tous libres des prescriptions de la Loi $(2,4)$. Indépendamment de leur origine juive ou païenne, ils ne sont justifiés que dans le Christ $(2,17)$. En Lui ils sont tous héritiers de la bénédiction d'Abraham $(3,14)$. Par la foi dans le Christ ils sont tous fils de Dieu $(3,26)$. Entre eux a disparu toute différence négative: tous ils sont un en Christ Jésus $(3,28)^{43}$.

Ga 5,6 fait penser à un autre passage de l' Épître aux Galates: «Car, ce qui importe, ce n'est ni la circoncision, ni l'incirconcision, mais la nouvelle création» (Ga 6,15) ${ }^{44}$. L'œuvre divine, l'œuvre du Christ sauve-transforme. Il y a dans le Christ, grâce à Lui, un renouvellement et l'accomplissement de la vie: ce qui est essentiel pour toute l'histoire (l'œuvre) de la créationrédemption, ce qui ne peut s'accomplir que grâce au bienveillant engagement divin ${ }^{45}$. C'est le Christ même qui aime, qui propage son amour. De cette façon, ensemble avec le Père et l'Esprit ( $c f$. Ga 1,1-5; 4,3-7; 6,8), Il unit, renouvelle, conduit vers l'eschatologique éternelle plénitude de la vie: «si quelqu'un est en Christ, il est une nouvelle créature. Le monde ancien est passé, voici qu'une réalité nouvelle est là» (2 Co 5,17). D'une certaine manière il s'agit donc de la participation actuelle déjà dans l'ici-bas. Aussi en affrontant des graves difficultés qu'on doit surmonter ( $c f$. par ex. Ga 1,6s) l'Apôtre croit et voit que l'homme, le chrétien est en communion d'amour et vie avec Dieu et avec ses sœurs et frères, que cette communion est largement ouverte aux suivantes créatures, que cette union se révèle aussi comme amour transformant de la part de l'homme ( $c f$. par ex. Ga 4,13s).

${ }^{43}$ Notons dans ce contexte encore une chose. Le seul texte parmi les passages de la Lettre aux Galates dans lesquels on rencontre les formules du type en Christô, qui ne concerne pas explicite en même temps les chrétiens d'origine juive et ceux d'origine païenne, c'est Ga 1,22. Mais est-ce que, quand St Paul écrit aux Galates, les chrétiens de Judée étaient uniquement d'origine juive?

${ }^{44}$ Dans certains manuscrits, en plus, en Ga 5,6 il y a en Christô lêsou (dans le Christ Jésus; cf. l'apparat critique).

${ }^{45}$ Cf. K.B. McCruden, Judgment and Life for the Lord: Occasion and Theology of Romans 14,1 - 15,13, in: Biblica 86(2005), p. 236; J.R. Levison, Creation and New Creation, in: Dictionary of Paul and His Letters. Editors: G.F. Hawthorne etc., Downers Grove-Leicester 1993, p. 189s; W. Misztal, Odnowienie i udzielenie petni życia w Świętym Duchu Ojca i Chrytusa. Studium na podstawie listów św. Pawła Apostoła, Kraków 2002, p. 443s. 


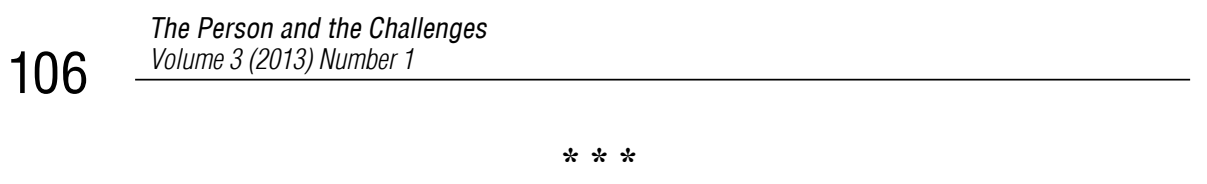

L'avènement du Christ marque, fait un des tournants les plus décisifs dans l'histoire de la création-rédemption. Il est vrai qu'il faut encore attendre que l'ordre ancien du péché et de la mort soit définitivement aboli, anéanti, qu'il cède d'une manière complète la place au Christ et à son règne ( $c f$. par ex. Rm $5,12 \mathrm{~s} ; 1$ Co 15,25s). D'autre part ce règne est déjà présent. Celui qui appartient au Christ et se trouve sous sa seigneurie, passe de l'ordre ancien à l'ordre nouveau établi par le Sauveur. D'une part il s'agit ici de quelque chose de ponctuel. Par la foi et le baptême l'homme scelle son adhésion au Christ et reçoit sa part à l'œuvre de la rédemption: à ce que le Sauveur a accompli en notre faveur ainsi qu'à ce qu'Il est en train d'accomplir pour nous à présent et à ce qu'Il accomplira encore. D'autre part doit-on voir ici quelque chose de continuel. C'est notre vie entière qui est nourie de la grâce, qui devient une succession de confirmation (ou de re-confirmation) de l'appartenance au Christ et à son domaine.

Les textes avec les formules du type dans le Christ (gr. en Christô) présentent notre vie d'ici bas comme une vie qui dépend tout à fait positivement du Sauveur, qui se déroule sous son vivifiant empire, sous ses auspices. Le Sauveur, Lui qui est le Seigneur (gr. kyrios) de toute la création, nous seconde tout au long de notre existence terrestre ( $c f$. par ex Ph 2,6s; 3,20s). St Paul insiste sur le fait qu'avec notre adhésion au Christ notre situation ici-bas change radicalement. Ce sont aussi nos jours d'ici-bas qui ont déjà été placés sous son empire, c'est-à-dire ils sont le chemin du salut, ils mènent vers la résurrection, vers l'eschatologique plénitude de la vie ( $c f$. par ex. 1 Co 15,21s).

\section{Bibliography}

Benoît XVI, Verbum Domini. Exhortation apostolique post-synodale aux évêques, au clergé, aux personnes consacrées et aux fidèles laïcs sur la parole de Dieu dans la vie et dans la mission de l'Eglise (30.09.2010).

Brown R.E., Que sait-on du Nouveau Testament? Présentation de P. Debergé, Paris 2000. Bultmann R., Theologie des Neuen Testaments, Tübingen 1980.

Catéchisme de l'Église Catholique, Paris 1992.

Chevallier M.-A., Souffle de Dieu. Le Saint-Esprit dans le Nouveau Testament, t. III Etudes. Avant-propos de J.-N. Aletti, Paris 1991.

Corsani B., Lettera ai Galati, Genova 1990. 
Descamps A., Le baptême, fondement de l'unité chrétienne, in: Battesimo e giustizia in Rom 6 e 8. A cura di L. De Lorenzi etc. Roma 1974, p. 203-234.

Dictionary of Paul and His Letters. Editors: G.F. Hawthorne etc., Downers GroveLeicester 1993.

Dognin P.-D., «La foi étant venue». l'Épître aux Galates, Bruxelles 2001.

Durrwell F.X., Ojciec. Bóg w swoim misterium, Kielce 2000.

Fitzmyer J.A., The Letter to the Galatians, in: The New Jerome Biblical Commentary. Edited by R.E. Brown S.S. etc., London 1992, p. 780-790.

Jean Paul II, Le progrès rapide. Lettre apostolique aux responsables des communications sociales (24.01.2005).

Légasse S., Paul apôtre. Essai de biographie critique. Deuxième édition revue, Paris 2000.

Marchel W., Abba. Père. La prière du Christ et des chrétiens. Etudes sur les origines et la signification de l'invocation à la divinité comme père avant et dans le Nouveau Testament, Rome 1971.

Misztal W., Duchowość chrześcijan w świetle listów Pawłowych: związki z Bogiem, Kraków 2010.

Penna R., Essere cristiani secondo Paolo, Casale Monferrato 1979.

Pindel R., W obronie prawdy Ewangelii. Analiza literacko-historyczna Ga 1,11-2,21, Kraków 2001.

Pitta A., Lettera ai Galati. Introduzione, versione, commento, Bologna 1996.

Preiss T., La vie en Christ. Préface de R. Mehl, Neuchâtel-Paris 1951.

Theologisches Wörterbuch zum Neuen Testament, éd. G. Kittel - G. Friedrich, t. I-IX, Stuttgart 1933-1973.

Vanni U., Duchowość Pawła, in: Historia duchowości. Tom II. Duchowość Nowego Testamentu, Kraków 2003, p. 145-185.

Vocabulaire de théologie biblique. Publié sous la direction de X. Léon-Dufour etc. Deuxième édition révisée et augmentée, Paris 1971.

Vouga F., La vérité de l'Évangile et la création nouvelle: l'apôtre Paul comme interprète de Jésus de Nazareth, in: Paul, une théologie en construction. Sous la direction de A. Dettwiler etc., Genève 2004, p. 343-361. 